University of Nebraska - Lincoln

DigitalCommons@University of Nebraska - Lincoln

7-15-1991

\title{
Construction of a quasiconserved quantity in the Henon-Heiles problem using a single set of variables
}

\author{
Paul Finkler \\ University of Nebraska-Lincoln, pfinkler1@unl.edu \\ C. Edward Jones \\ University of Nebraska-Lincoln \\ Glenn A. Sowell \\ University of Nebraska-Lincoln
}

Follow this and additional works at: https://digitalcommons.unl.edu/physicsfinkler

Part of the Physics Commons

Finkler, Paul; Jones, C. Edward; and Sowell, Glenn A., "Construction of a quasiconserved quantity in the Henon-Heiles problem using a single set of variables" (1991). Paul Finkler Papers. 4.

https://digitalcommons.unl.edu/physicsfinkler/4

This Article is brought to you for free and open access by the Research Papers in Physics and Astronomy at DigitalCommons@University of Nebraska - Lincoln. It has been accepted for inclusion in Paul Finkler Papers by an authorized administrator of DigitalCommons@University of Nebraska - Lincoln. 


\title{
Construction of a quasiconserved quantity in the Hénon-Heiles problem using a single set of variables
}

\author{
Paul Finkler, C. Edward Jones, and Glenn A. Sowell \\ Department of Physics and Astronomy, University of Nebraska-Lincoln, Lincoln, Nebraska 68588-0111
}

(Received 10 May 1990; revised manuscript received 27 March 1991)

\begin{abstract}
The problem of finding the coefficients of a simple series expansion for a quasiconserved quantity $K$ for the Hénon-Heiles Hamiltonian $H$ using a single set of variables is solved. In the past, this type of approach has been problematic because the solution to the equations determining the coefficients in the expansion is not unique. As a result, the existence of a consistent expression for $K$ to all orders had not previously been established. We show how to deal with this arbitrariness in the expansion coefficients for $K$ in a consistent way. Due to this arbitrariness, we find a class of expansions for $K$, in contrast to the single unique expansion for $K$ generated by the normal-form approach of Gustavson [Astron. J. 71, 670 (1966)]. It may be possible to devise a criterion for deciding which one of our expansions is "optimally convergent," although we do not deal with this question here. We proceed by introducing a single set of dynamic variables that have simple symmetry properties and that also "diagonalize" the problem of finding the coefficients of $K$. No canonical transformations are required. A straightforward constructive procedure is given for generating the power series to any order for quantities having the symmetry of the Hamiltonian that are formally conserved. This leads to a very practical method for calculating a quasiconserved quantity in the Hénon-Heiles problem. A comparison is made through several orders of the terms generated by this approach and those generated in the original Gustavson expansion in normal form.
\end{abstract}

\section{INTRODUCTION}

For a number of years, the Hénon-Heiles potential [1] has served as a useful laboratory for studying the properties of chaotic motion and nonlinear dynamics. Numerical studies indicate that some trajectories in the HénonHeiles potential are chaotic, while others apparently are not (for a recent discussion of this see, e.g., Ref. [2], which will hereafter be referred to as FJS1). Chaotic trajectories produce Poincaré sections which are area filling, whereas nonchaotic trajectories produce one-dimensional closed-curve Poincaré sections [1,2].

Of particular interest has been the question of whether a second conserved quantity exists in the Hénon-Heiles problem in addition to the energy. Closed-curve Poincaré sections suggest the presence of such a conserved quantity or first integral. Area-filling Poincare sections suggest the absence of such a quantity. Approximately conserved quantities have been found and are expressed in power-series expansions in terms of the coordinates and velocities $[2,3]$. Such quantities were originally studied by Gustavson [3], whose method brought the Hamiltonian into normal form by a succession of canonical transformations. He truncated the series after five orders and found that the resulting expression was, indeed, approximately conserved. More recently Shirts and Reinhardt [4] have calculated ten terms in the Gustavson normal-form expansion and have used Padé approximants to study the convergence of the series.

The so-called Gustavson-Birkhoff normal formal technique has also been used extensively as the basis of a method of quantizing a system consisting of harmonic os- cillators subject to a nonlinear perturbation [5]. Some of these authors use semiclassical quantization techniques and others use algebraic methods involving annihilation and creation operators. Newer quantization methods have gone beyond the normal form approach to embody techniques based on Lie operators [6]. The aim of such programs is to simplify the form of the Hamiltonian by making a succession of canonical transformationswhich in the Lie operator formalism, as opposed to the normal form method, do not have to be inverted. Once the Hamiltonian is simplified, e.g., written in terms of action-angle variables, the energy can be quantized. One by-product of the methods described above is the identification of quantities that are formally constants of the motion or invariants of the Hamiltonian, which can be written in simple form in terms of the new transformed variables.

In the current paper we do not deal with the quantization question, but are interested only in the construction of approximately conserved quantities. These quantities are expressed as power series in the coordinates and momenta, as in the original work of Gustavson, but we do not perform a succession of canonical transformations to get this series. This approach is an extension of the one introduced in FJS1. The method there can be briefly summarized by saying that a power series for a constant of the motion $K$ with unknown coefficients is introduced. Then we require the Poisson bracket $[K, H]$ be identically zero, where $H$ is the Hénon-Heiles Hamiltonian, and solve for the expansion coefficients of $K$. This method is not new, but had never been successfully employed in the past because in the resonant case-such as Hénon- 
Heiles - the harmonic-oscillator frequencies are commensurate. In this case, the equations determining the coefficients of $K$ have nonunique solutions. Furthermore, if this nonuniqueness is removed in some arbitrary manner, the equations for higher-order coefficients will in general have no solutions. On the other hand, the Gustavson procedure gives a well-defined algorithm for consistently finding unique expansion coefficients for $K$ to all orders. In FJS1 we developed a procedure for determining the coefficients by eliminating the arbitrariness in a manner that appeared to be consistent, although we lacked a proof that our procedure could be continued indefinitely to all orders. We utilized in a fundamental way the particular discrete symmetries of the HénonHeiles potential to reduce the total number of terms that needed to be considered at each order. With this technique, we generated twelve orders in the conserved quantity $K$, exceeding the number of orders obtained by both Gustavson [3] and Shirts and Reinhardt [4].

General considerations (see, e.g., the work of Ziglin [7]) indicate that such power series for conserved quantities are not expected to converge in general. However, we are not aware of any general proof that would rule out the possibility that certain series for a conserved quantity might converge along some nonchaotic trajectories while failing to converge along chaotic trajectories or portions thereof. The results in FJS1 can be taken as numerical support for such a conjecture since we see there no clear evidence of divergence of the series along clearly nonchaotic trajectories, but do see such evidence of divergence along chaotic trajectories (see also Ref. [4]).

In the present paper, we define a new set of variables whose symmetry properties are clearly evident and that also "diagonalize" the problem. Thus the symmetry properties of the conserved quantity are easy to analyze and the matrix inversion process required in FJS1 becomes unnecessary. The problem of the arbitrariness of the coefficients mentioned earlier is completely solved here. It is shown how that arbitrariness can be removed in a consistent way that guarantees the procedure can be carried to all orders. Of particular interest is the fact that we are able to discover exactly how to vary the arbitrariness in the series for $K$ in a consistent way. This will hopefully lead in the future to a procedure for finding an optimally convergent series for $K$. The typical normal form treatments lead to a unique $K$, with no discussion of the arbitrariness.

Our method gives an efficient and straightforward procedure for generating a power series expression for a conserved quantity in the Hénon-Heiles problem. We are able to calculate at least twice as many orders as in FJS1 without undue strain on standard computer facilities. We emphasize that only one set of variables is used throughout so no inversions of transformations of the type required in other methods are needed. Using our technique we hope to gain further insight into the questions raised in the preceding paragraphs on the possible relationship between chaos and the divergence or nonconvergence of the series.

This method for constructing the series for the conserved quantity is simple and constructive; only straight- forward differentiations are required, which can be readily carried out by a computer algebra program. Early indications are that the number of terms generated at each order appear to be surprisingly small and manageable. We show that the procedure can be carried out to indefinitely high order, a proof which was missing in our earlier method (FJS1).

In this paper, we shall concern ourselves principally with explaining the method; we will report elsewhere detailed numerical results. However, we do provide comparison in Sec. VIII between the expansion coefficients given by our method and those given by the normal-form approach of Gustavson for orders of $K$ from fourth to sixth.

\section{DEFINITION OF THE VARIABLES AND THE EQUATIONS OF MOTION}

The Hénon-Heiles problem involves motion in two dimensions with the following kinetic energy $T$ and potential energy $U$ :

$$
\begin{aligned}
& T=\frac{1}{2}\left(\dot{x}^{2}+\dot{y}^{2}\right), \\
& U=\frac{1}{2}\left(x^{2}+y^{2}\right)+x^{2} y-\frac{1}{3} y^{3} .
\end{aligned}
$$

In phase space we have four variables, two coordinates, and two momenta which here are simply the velocities. We now transform to a set of four complex variables which, as we shall see, are particularly well suited to this problem and have simple symmetry properties. These variables are defined as follows:

$$
\begin{aligned}
& \beta=(x+i y)+i(\dot{x}+i \dot{y}), \\
& \beta^{*}=(x-i y)+i(\dot{x}-i \dot{y}), \\
& \bar{\beta}=(x+i y)-i(\dot{x}+i \dot{y}), \\
& \bar{\beta}^{*}=(x-i y)-i(\dot{x}-i \dot{y}) .
\end{aligned}
$$

We note that (2.3) and (2.6) are complex conjugates of one another as are (2.4) and (2.5).

To facilitate our later discussions, it will be convenient to break up the total energy $E$ into quadratic and cubic terms as follows:

$$
E=T+U=E_{2}+E_{3},
$$

where

$$
\begin{aligned}
& E_{2}=\frac{\bar{\beta}^{*} \beta+\bar{\beta} \beta^{*}}{4}, \\
& E_{3}=\frac{(\beta+\bar{\beta})^{3}-\left(\beta^{*}+\bar{\beta}^{*}\right)^{3}}{48 i} .
\end{aligned}
$$

The expression $E_{2}$ represents the energy of a symmetric harmonic oscillator in two dimensions, while $E_{3}$ is just the cubic or anharmonic part of the potential. Both expressions (2.8) and (2.9) are real as they should be.

We also record here the equations of motion satisfied by the $\beta$ variables:

$$
\dot{\beta}=-i \beta+\frac{1}{4}\left(\beta^{*}+\bar{\beta}^{*}\right)^{2},
$$




$$
\begin{aligned}
& \dot{\bar{\beta}}^{*}=i \bar{\beta}^{*}+\frac{1}{4}(\beta+\bar{\beta})^{2}, \\
& \dot{\bar{\beta}}=i \bar{\beta}-\frac{1}{4}\left(\beta^{*}+\bar{\beta}^{*}\right)^{2}, \\
& \dot{\beta}^{*}=-i \beta^{*}-\frac{1}{4}(\beta+\bar{\beta})^{2} .
\end{aligned}
$$

\section{SYMMETRIES}

A study of the form of the Hénon-Heiles potential (2.2) shows that several important symmetries are present in the problem. A knowledge of these will be extremely important in developing our method for determining a conserved quantity for this potential. The symmetries are these: (1) time reversal; (2) reflection through the origin in the $x$ variable (i.e., $x$ goes to $-x$ ); and (3) invariance under rotations in the $x-y$ plane by multiples of $120^{\circ}$. Symmetries (2) and (3) mean that the potential is invariant under the group of transformations which maps the equilateral triangle into itself. The symmetries just mentioned are easily established once we know how the $\beta$ variables transform under time reversal, $x$ reflection, and general rotations in the $x-y$ plane. The $\beta$ variables are readily found to have the following transformation properties:

$\beta \leftrightarrow \bar{\beta}, \quad \beta^{*} \leftrightarrow \bar{\beta}^{*} \quad$ (time reversal) ,

$\beta \leftrightarrow-\beta^{*}, \quad \bar{\beta} \leftrightarrow-\bar{\beta}^{*} \quad(x$ reflection $)$,

$\beta \rightarrow \beta \exp (i \phi), \quad \beta^{*} \rightarrow \beta^{*} \exp (-i \phi)$,

$\bar{\beta} \rightarrow \bar{\beta} \exp (i \phi), \quad \bar{\beta}^{*} \rightarrow \bar{\beta}^{*} \exp (-i \phi), \quad($ rotation by $\phi)$.

We conclude from (3.3) that any monomial constructed of powers of the four $\beta$ variables will transform under rotations in a manner which is determined by the difference between the sum of the exponents of all unstarred $\beta$ variables and the sum of the exponents of all starred $\beta$ variables. For example, if this difference is zero, the monomial is rotationally invariant. This is the case, e.g., for $E_{2}$ in (2.8), which consists of the sum of two monomials, each a product of one starred and one unstarred $\beta$ variable. By contrast, $E_{3}$ in (2.9) consists of a sum of thirdorder monomials, each a product of three $\beta$ variables, all of which are either starred or unstarred, which will transform under rotations either as $\exp (-i 3 \phi)$ or $\exp (i 3 \phi)$. It follows that the Hénon-Heiles system is invariant under rotations by angles which are integer multiples of $120^{\circ}$ as indicated earlier. One can also verify that $E_{2}$ and $E_{3}$ are both invariant under the time-reversal transformation (3.1) and $x$ reflection (3.2).

\section{EQUATIONS FOR A CONSERVED QUANTITY}

In this section, we begin developing the equations that must be satisfied by a conserved quantity which we call $K$. We shall ultimately show that a particular power series can be constructed for such a $K$ that is invariant under the symmetries in the problem [8]. Also $K$ must not be merely a function of the energy and it must have a vanishing total time derivative:

$$
\dot{K}=0 \text {. }
$$

Using the equations of motion (2.10)-(2.13) above, we can express the time derivative of $K$ in terms of derivatives with respect to the $\beta$ variables. We find

$$
\dot{K}=-D_{0} K+D_{1} K=0,
$$

where $D_{0}$ and $D_{1}$ are differential operators defined as follows:

$$
\begin{aligned}
D_{0}=i & {\left[\beta \frac{\partial}{\partial \beta}+\beta^{*} \frac{\partial}{\partial \beta^{*}}-\bar{\beta} \frac{\partial}{\partial \bar{\beta}}-\bar{\beta}^{*} \frac{\partial}{\partial \bar{\beta}^{*}}\right], } \\
D_{1}=\frac{1}{4} & {\left[\left(\beta^{*}+\bar{\beta}^{*}\right)^{2} \frac{\partial}{\partial \beta}+(\beta+\bar{\beta})^{2} \frac{\partial}{\partial \bar{\beta}^{*}}\right.} \\
& \left.-(\beta+\bar{\beta})^{2} \frac{\partial}{\partial \beta^{*}}-\left(\beta^{*}+\bar{\beta}^{*}\right)^{2} \frac{\partial}{\partial \bar{\beta}}\right] .
\end{aligned}
$$

We note that $D_{0}$ and $D_{1}$ transform as follows under the symmetry transformations (3.1)-(3.3).

(i) $D_{0}$ is antisymmetric under time reversal, symmetric under $x$ reflection, and invariant under continuous rotations.

(ii) $D_{1}$ is antisymmetric under time reversal, symmetric under $x$ reflection, and invariant under discrete rotations by integer multiples of $120^{\circ}$.

We now assume $K$ to be written as a power series in the four $\beta$ variables:

$$
K\left(\beta, \beta^{*}, \bar{\beta}, \bar{\beta}^{*}\right)=\sum_{n=n_{0}}^{\infty} K_{n},
$$

where $K_{n}$ is an $n$th order term in the $\beta$ variables and $n_{0}>0$. In order for the time derivative of $K$ to vanish identically the $K_{n}$ must by (4.2) satisfy the following equations:

$$
\begin{aligned}
& D_{0} K_{n_{0}}=0, \\
& D_{0} K_{n+1}=D_{1} K_{n} .
\end{aligned}
$$

Equations (4.6) and (4.7) follow from the fact that the operator $D_{0}$ leaves the order of a given term unchanged, while $D_{1}$ raises the order by 1 .

The essential point of this paper is to formulate a method for finding a solution to the infinite sequence of Eqs. (4.6) and (4.7) and hence to generate the series (4.5) for $K$. Superficially, a quite straightforward approach appears to be as follows: first solve (4.6), insert this solution on the right-hand side of (4.7), and then solve for the term of order $n_{0}+1$. Continuing the iterative process would seem, in principle, to generate all orders. The problem is that there exist solutions $H$ to the homogeneous equation:

$$
D_{0} H=0
$$

This means that in general (4.7) has no solution for $K_{n+1}$ unless the right-hand side of (4.7) is "orthogonal" to all homogeneous solutions $H$, as we explain later. Dealing with the presence of homogeneous solutions is the key technical problem in generating the series (4.5). In the 
following section we discuss the $D_{0}$ operator and the solutions of the homogeneous equation (4.8).

\section{PROPERTIES OF THE $D_{0}$ OPERATOR AND SOLUTIONS TO THE HOMOGENEOUS EQUATION}

Fortunately, it is very simple to completely characterize all the solutions to the homogeneous equation (4.8). Since we shall work entirely in a function space spanned by monomials in the $\beta$ variables, we begin by giving the effect of $D_{0}$ on a general monomial in this space. From (4.3) we find

$$
D_{0} M_{p q r s}=i(p+q-r-s) M_{p q r s},
$$

where

$$
M_{p q r s}=\beta^{p} \beta^{* q} \bar{\beta}^{r} \bar{\beta}^{* s} .
$$

We see that the operator $D_{0}$ is diagonal in the space of the monomials in the $\beta$ variables. The solutions to the homogeneous equation (4.8) in this space of monomials are then completely specified by the condition

$$
p+q-r-s=0 \text {. }
$$

We note that the inverse operator $D_{0}^{-1}$ can clearly be given a unique well-defined meaning when acting on any of the monomials for which $(p+q+r-s)$ is nonvanishing, viz.,

$$
D_{0}^{-1} M_{p q r s}=\frac{1}{i(p+q-r-s)} M_{p q r s} .
$$

Let us now return to a discussion of the homogeneous solutions that satisfy (5.3). We see that any monomial will be a homogeneous solution if the total exponent of all the barred $\beta$ variables is equal to the total exponent of all the unbarred variables. Thus all homogeneous solutions are sums of even-order monomials. No combination of odd-order monomials can be a homogeneous solution. This means that (4.7) always possesses a unique solution whenever $(n+1)$ is odd and this solution can be easily determined by applying (5.4).

We now wish to discuss homogeneous solutions that have special symmetry properties. First, a monomial homogeneous solution will have continuous rotational symmetry if

$$
p+r=q+s \text {. }
$$

This together with (5.3) implies that $p=s$ and $q=r$. Designating a general monomial solution to the homogeneous equation with continuous rotational symmetry by $H_{p q}^{C}$ we have the result

$$
H_{p q}^{C}=\beta^{p} \beta^{* q} \bar{\beta}^{q} \bar{\beta}^{* p},
$$

which has order $n=2(p+q)$. From (3.1) and (3.2) we see that $H^{C}$ is invariant under combined time reversal and $x$-reflection transformations, but not generally under the individual transformations. However, by adding two of these monomials we can always form an expression, denoted $H_{p q}^{++C}$, which is invariant separately under time reversal and $x$ reflection:

$$
H_{p q}^{++C}=\beta^{p} \beta^{* q} \bar{\beta}^{q} \bar{\beta}^{* p}+\beta^{q} \beta^{* p} \bar{\beta}^{p} \bar{\beta}^{* q} .
$$

In our notation, we take the first index of the superscript as plus or minus to indicate evenness or oddness under time reversal, the second index to indicate the same for $x$ reflection, and the third index will be $C$ or $D$ indicating continuous rotational symmetry or discrete rotational symmetry (i.e., rotations by integers multiples of $120^{\circ}$ ). In the special case where $p=q$, the two terms in (5.7) are the same and that single monomial possesses the full symmetry. By inserting a minus sign between the two terms on the right-hand side of (5.7), we arrive at a homogeneous solution with $(--C)$ symmetry:

$$
H_{p q}^{-}{ }^{-C}=\beta^{p} \beta^{* q} \bar{\beta}^{q} \bar{\beta}^{* p}-\beta^{q} \beta^{* p} \bar{\beta}^{p} \bar{\beta}^{* q} .
$$

We see that it is impossible to construct homogeneous solutions with $(+-C)$ or $(-+C)$ symmetries.

The simplest example of (5.7) occurs at order two with $p=1, q=0$, and the result is a multiple of $E_{2}$ :

$$
H_{10}^{++C}=4 E_{2} \text {. }
$$

The simplest example of (5.8), again with $p=1, q=0$, is found to be a multiple of the angular momentum $(x \dot{y}-y \dot{x})$ denoted $L_{2}$ :

$$
H_{10}^{-}-C=4 L_{2} \text {. }
$$

It will be useful for what follows later to record here two special classes of homogeneous solutions that have discrete rotational symmetry and that occur at orders $6 \mathrm{~m}$ where $m$ is a positive integer:

$$
h_{6 m}^{++D}=\left[(\beta \bar{\beta})^{3 m}+\left(\beta^{*} \bar{\beta}^{*}\right)^{3 m}\right]
$$

and

$$
h_{6 m}^{+-D}=\left[(\beta \bar{\beta})^{3 m}-\left(\beta^{*} \bar{\beta}^{*}\right)^{3 m}\right], \quad m=1,2,3, \cdots .
$$

\section{OPERATOR THEOREMS}

In Sec. $\mathrm{V}$ we completely characterized all the homogeneous solutions to (4.8) in the space of monomials in the $\beta$ variables. We also identified classes of homogeneous solutions possessing certain symmetry properties. In this section we begin to apply those results to construct solutions of (4.6) and (4.7). However, it will be useful first to give several theorems satisfied by the operators in the problem relating to their action on certain of the homogeneous solutions to (4.8) discussed in the preceding section.

First we observe that the lowest order $n_{0}$ in the series for $K$ must be even because we have seen in the preceding section that solutions to (4.6) only exist for even order. We can then solve (4.7) immediately for $K_{n_{0}+1}$; the unique solution is

$$
K_{n_{0}+1}=D_{0}^{-1} D_{1} K_{n_{0}} \text {. }
$$

We note that $D_{1} K_{n_{0}}$ is of order $n_{0}+1$ which is odd and therefore $D_{0}^{-1}$ on this expression is well defined by (5.4). The resulting $K_{n_{0}+1}$ will consist of a sum of monomials, 
each of order $n_{0}+1$. Since we are at odd order, none of the denominators in (5.4) vanish. The next step is to determine $K_{n_{0}+2}$, which from (4.7) satisfies the equation

$$
D_{0} K_{n_{0}+2}=D_{1} D_{0}^{-1} D_{1} K_{n_{0}}
$$

The right-hand side of (6.2) is a sum of monomials each of even order $\left(n_{0}+2\right)$. The equation will have no solution if any of these monomials is a homogeneous solution [i.e., with exponents satisfying (5.3)] because the inverse operator $D_{0}^{-1}$ [see (5.4)] would not be defined in this case.

The problem of determining whether an expression such as the right-hand side of (6.2) contains any terms which are homogeneous solutions will be a recurring one. It is convenient therefore to introduce an operator $\Omega$ (to be used on monomials of even order only), which is defined as follows:

$$
\Omega=\left(1-D_{0}^{-1} D_{0}\right) D_{1} D_{0}^{-1} D_{1} \text {. }
$$

The term in parentheses on the right-hand side of (6.3) is just the projection operator for homogeneous solutions. From our discussion in Sec. IV following (4.4), we can conclude that $\Omega$ has the symmetry $(-+D)$.

Equation (6.2) will then have a solution (actually many solutions) if and only if

$$
\Omega K_{n_{0}}=0 \text {. }
$$

In other words, the right-hand side of (6.2) will include no monomials satisfying (5.3) if (6.4) is satisfied (i.e., be "orthogonal" to all homogeneous solutions).

We now state three important theorems involving the $\Omega$ operator. The proofs of these theorems will be sketched in the appendix.

Theorem I:

$$
\Omega H_{p q}^{++C}=0 \text {. }
$$

Theorem I can be proved by direct calculation using (5.7) and the definitions of the differential operators.

From Theorem I it follows that if $K_{n_{0}}$ has $(++C)$ symmetry, (6.2) will have a solution. In constructing $K$, we shall therefore pick the lowest order term $K_{n_{0}}$ to have this symmetry, thus guaranteeing a solution to (6.2).

Theorem II:

$$
\Omega\left(H_{1} H_{2}\right)=\left(\Omega H_{1}\right) H_{2}+H_{1}\left(\Omega H_{2}\right),
$$

where $H_{1}$ and $H_{2}$ are both solutions of the homogeneous equation (4.8).

Theorem III:

$$
\Omega h_{6 m}^{++D}=\Omega_{0} m h_{6 m}^{+-D} L_{2}
$$

where $m=1,2,3, \cdots$ and $\Omega_{0}=-7 i$.

Since $\Omega$ is antisymmetric under time reversal, the lefthand side of (6.7) must have the symmetry $(-+D)$. Since $L_{2}$ has the symmetry $(--C)$, we see that the right-hand side of (6.7) also has the symmetry $(-+D)$ as it must.

The three theorems discussed in this section will be extremely useful in the following section where we describe in detail the construction of a unique series for $K$ possessing the full symmetry of the problem.

\section{PROCEDURE FOR CONSTRUCTION $K$}

We are now in a position to give systematic steps for constructing a series for the conserved quantity $K$. As we have seen, the lowest order $n_{0}$ must be even for $K_{n_{0}}$ to be a solution of the homogeneous equation (4.6). We shall also assume that $K_{n_{0}}$ has the symmetry $(++C)$. As explained in the preceding section, this will ensure that (6.2) has a solution for the order $\left(n_{0}+2\right)$ term. The order $\left(n_{0}+1\right)$ term is given by (6.1) and has the symmetry $(++D)$.

If we take for the lowest-order term $n_{0}=2$, we find $K_{2}=E_{2}$, since this is the only term with the symmetry $(++C)$. The third-order term generated by (6.1) will then just be $E_{3}$. The series will then terminate and $K$ becomes just the total energy unless we introduce a nontrivial solution to (4.6) at fourth order (or any higher even order). So to construct a $K$ that is not just a function of the energy, we take the lowest order to be 4 . At order 4 there are two linearly independent homogeneous solutions with symmetry $(++C)$ corresponding in (5.7) to $p=1, q=1$, and $p=2, q=0$. These correspond to linear combinations to $E_{2}^{2}$ and $L_{2}^{2}$.

We shall designate our starting point by $K_{n_{0}}^{++C}$, where we expect to take $n_{0}=4$. Order $n_{0}+1$ is determined uniquely by (6.1) using (5.4). All odd orders are determined in this straightforward way and will have the symmetry $(++D)$, if the preceding even-order term has that symmetry.

From Theorem I, we know that $K_{n_{0}}^{++C}$ satisfies (6.4):

$$
\Omega K_{n_{0}}^{++C}=0 .
$$

Thus a term of order $\left(n_{0}+2\right)$ which is a particular solution of (4.7) is given by

$$
\begin{aligned}
K_{n_{0}+2}^{++D}(\text { non }) & =D_{0}^{-1} D_{1} K_{n_{0}+1}^{++D} \\
& =D_{0}^{-1}\left(D_{1} D_{0}^{-1} D_{1} K_{n_{0}}^{++C}\right) .
\end{aligned}
$$

Since (7.1) is satisfied, we know that the expression in parentheses on the right-hand side of (7.2) contains no monomials which are homogeneous solutions and therefore the inverse of $D_{0}$ will be defined on this quantity using (5.4). The argument (non) in (7.2) indicates that this is a unique particular solution to (4.7) containing only nonhomogeneous solutions to that equation, i.e., no monomials occur on the right-hand side of (7.2) satisfying the homogeneous equation (4.8) and, thence, (5.3). That (7.2) has $(++D)$ symmetry follows from the fact that $D_{0}^{-1} D_{1}$ has this symmetry as can be seen from our discussion in Sec. IV.

Adding to (7.2) any solution to the homogeneous equation (4.8) of order $\left(n_{0}+2\right)$ also gives a solution to (4.7). Although it is tempting to try to use just the unique particular solution (7.2), we cannot then, in general, find a solution to (4.7) at the next even order $\left(n_{0}+4\right)$. In fact, 
we shall see that a certain homogeneous solution must be added to (7.2) (and to the nonhomogeneous solution at each even order) in order to continue the process and find solutions to (4.7) for all orders. Fortunately, the homogeneous solution which we are required to add at each even order is easy to determine in the $\beta$ variable formalism, as we shall see. After adding the required homogeneous solution just mentioned, we can also add arbitrary amounts of homogeneous solutions with $(++C)$ symmetry leading to a whole class of expansions for $K$ as we discuss later.

The difficulty with taking (7.2) as the complete term at order $\left(n_{0}+2\right)$ is that

$$
\Omega K_{n_{E}}^{++D}(\text { non }) \neq 0,
$$

where for now $n_{E}=\left(n_{0}+2\right)$. This means that (4.7) will not possess a solution for order $\left(n_{E}+2\right)=\left(n_{0}+4\right)$. Our procedure is to add to (7.2) a solution of the homogeneous equation with $(++D)$ symmetry giving a total term of order $n_{E}=\left(n_{0}+2\right)$ of the form

$$
K_{n_{E}}^{++D}=K_{n_{E}}^{++D}(\text { non })+H_{n_{E}}^{++D},
$$

where $H_{n_{E}}^{++D}$ is a homogeneous solution of order $n_{E}$ with symmetry $(++D)$ and

$$
\Omega K_{n_{E}}^{++D}=0 \text {. }
$$

It is not obvious that a term $H_{n_{E}}^{++D}$ can be found which will lead to (7.5). With the help of symmetry arguments, we shall be able to show that such a term can always be found. The crucial problem we wish to solvenamely, constructing a term of order $n_{E}$ which satisfies (7.5)-occurs at every even order $n_{E}$ in the series for which $n_{E}>n_{0}$. We have introduced the general even in$\operatorname{dex} n_{E}$ so that we can treat the problem at all even orders, not just at $n_{E}=\left(n_{0}+2\right)$. Once this problem is solved in general, it is a straightforward process to construct the series for $K$ to all orders.

In Sec. VI we noted that the operator $\Omega$ has the transformation property $(-+D)$. This means that

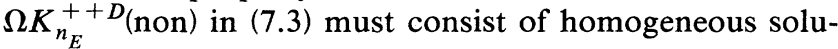
tions having the symmetry $(-+D)$ or possibly $(-+C)$. However, as indicated in Sec. V, terms with $(-+C)$ symmetry are impossible for homogeneous solutions possessing continuous rotational symmetry. Thus $\Omega K_{n_{E}}^{++D}$ (non) must be a homogeneous solution of (4.8) of even order $\left(n_{E}+2\right)$ having $(-+D)$ symmetry.

To proceed, let us define $m_{0}$ as the largest multiple of 6 contained in $\left(n_{E}\right)$, i.e.,

$$
m_{0}=\operatorname{int}\left(n_{E} / 6\right) \text {, }
$$

where int represents the integer part. Based on the fact that $\Omega K_{n_{E}}^{++D}$ (non) must have $(-+D)$ symmetry, it is possible to express it as a unique sum of products of terms of the type introduced in Sec. V as follows:

$$
\Omega K_{n_{E}}^{++D}(\text { non })=L_{2}^{--C} \sum_{m=1}^{m_{0}} h_{6 m}^{+-D} h_{n_{E}}^{++C}+C
$$

where we have added a superscript to the angular momentum $L_{2}$ making its symmetry properties explicit. It can be shown that any term with $(-+D)$ symmetry has an expansion of the type given by the right-hand side of (7.7). The $h_{n}^{++C}$ terms in (7.7) are homogeneous solutions of order $n$ having $(++C)$ symmetry. These terms are determined from the expansion (7.7) itself, unlike the $L_{2}$ and $h_{6 m}^{+-D}$ functions which have been previously specified. In general, they will consist of a linear combination of terms of the form of (5.7). It is clear from (5.3) that products of solutions of (4.8) also satisfy (4.8). Thus each term in (7.7) is a solution of the homogeneous equation because it is made up of a product of three factors each of which is a homogeneous solution.

We are now in a position to construct an expression for the function $H_{n_{E}}^{++D}$ in (7.4) which will satisfy (7.5) ensuring that a solution for $K$ of order $\left(n_{E}+2\right)$ exists:

$$
H_{n_{E}}^{++D}=-\frac{1}{\Omega_{0}} \sum_{m=1}^{m_{0}} \frac{h_{6 m}^{++D} h_{n_{E}-6 m}^{++C}}{m}
$$

where the functions $h_{n}^{++C}$ are those in (7.7) and the $h^{++D}$ terms are given by $(5.12)$.

Applying the operator $\Omega$ to (7.8), we can use the chain rule Theorem II, since each factor in (7.8) is a solution to the homogeneous equation. By Theorem I, we conclude that the only terms that survive are those where $\Omega$ operates on the $h^{++D}$ factors. By Theorem III, we then see that the operation of $\Omega$ on (7.8) produces the negative of (7.7). Using the expression for $H_{n_{E}}^{++D}$ given by (7.8) in (7.4), we determine $K_{n_{E}}^{++D}$, which is now guaranteed to satisfy (7.5). The odd order term $K_{n_{E}+1}^{++D}$ is gotten as indicated earlier from (6.1) with $n_{0}=n_{E}$ using (5.4). We then start the process over again by determining $K_{n_{E}}^{++2}$ (non) from $K_{n_{E}}^{++D}$ as in (7.2):

$$
K_{n_{E}+2}^{++D}(\text { non })=\left(D_{0}^{-1} D_{1}\right)^{2} K_{n_{E}}^{++D}
$$

We thus have a simple constructive procedure for determining each term in the power series (4.5) with $K$ having the complete $(++D)$ symmetry of the problem. This process determines a unique $K$ from a given starting term $K_{n_{0}}^{++C}$

We are free, of course, to add on other terms to (7.8) which are solutions to the homogeneous equations with $(++C)$ symmetry. Because of Theorem I, (7.5) will continue to be satisfied and an infinite class of $K$ expansions can be generated. When this freedom of adding homogeneous solutions with $(++C)$ symmetry is combined with all possible starting points for the expansion, we believe the resulting class of $K$ expansions includes every possible positive-exponent power-series expansion of a formally conserved quantity. The members of this class are, of course, not all independent. In general, we expect any one member of this class to be expressible as a function of the total energy and any other member of the class. 


\section{COMPARISON WITH THE NORMAL-FORM APPROACH}

In this section we provide a comparison of our method with the original normal form approach of Gustavson [3] for the first few orders in the conserved quantity $K$. In Gustavson's method, there is no ambiguity in determining the series for the conserved quantity. In terms of the canonical variables $\xi_{i}$ and $\eta_{i}$, which result from a succession of canonical transformations that bring the Hamiltonian into normal form, the conserved quantity $I^{(2)}$ for the Hénon-Heiles problem takes the form of a simple superposition of two harmonic oscillators:

$$
I^{(2)}=\sum_{i=1}^{2} \frac{1}{2}\left(\xi_{i}^{2}+\eta_{i}^{2}\right) .
$$

When one expresses (8.1) in terms of the original variables given in Sec. II, we will have an infinite power series, the first two orders of which agree exactly with the total energy $T+U$ given in (2.1) and (2.2). It is therefore natural to deal with a conserved quantity $K$, which is the difference between $I^{(2)}$ and the total energy. Thus $K$, whose first nonvanishing order in the original variables is 4 , is defined by

$$
K=T+U-I^{(2)} \text {. }
$$

From the results of Gustavson [3], the first nonvanishing order of $K$, which we designate $K_{4}$, is found to be

$$
K_{4}=\frac{1}{96} \beta^{2} \bar{\beta}^{* 2}+\frac{1}{96} \beta^{* 2} \bar{\beta}^{2}-\frac{1}{8} \beta \beta^{*} \bar{\beta} \bar{\beta}^{*},
$$

where we have written $K_{4}$ in terms of the $\beta$ variables.

Starting with (8.3) we have followed the procedure outlined in Sec. VII for constructing $K$ and have generated $K_{5}$ and $K_{6}$, which are given in the first column of Table I labeled under the heading FJS. The expression for $K_{5}$ is determined uniquely from (6.1). We then determine $K_{6}^{++D}$ (non) from (7.2) and find $H_{0}^{++D}$ using (7.7) and (7.8). In (7.8), clearly only the $m=1$ term contributes. We thus arrive at the sixth-order term as in (7.4):

$$
K_{6}^{++D}=K_{6}^{++D}(\text { non })+H_{6}^{++D} .
$$

As in Sec. VII, $K_{6}^{++D}$ includes no homogeneous $(++C)$ terms and satisfies (7.5) so that the series for $K$ will be certain to continue through eight order.

The Gustavson normal-form series [3] for orders four through six is given in the second column of Table $I$, and in the third column the difference between the first two columns. We see that in fifth order the two procedures give the same result, whereas in sixth order, the Gustavson approach gives four extra terms which are shown in column 3. By comparing these terms with (5.7), we see that they are, indeed, $(++C)$ homogeneous terms as expected. As we have seen, these additional $(++C)$ homogeneous terms in the Gustavson expression are not

\begin{tabular}{|c|c|c|c|}
\hline Order & FJS & Gustavson & Difference \\
\hline 4 & $\frac{1}{96} \beta^{2} \bar{\beta}^{* 2}-\frac{1}{8} \beta \bar{\beta}^{*} \bar{\beta} \beta^{*}+\frac{1}{96} \bar{\beta}^{2} \beta^{* 2}$ & $\frac{1}{96} \beta^{2} \bar{\beta}^{* 2}-\frac{1}{8} \beta \bar{\beta}^{*} \bar{\beta} \beta^{*}+\frac{1}{96} \bar{\beta}^{2} \beta^{* 2}$ & 0 \\
\hline 5 & $\begin{array}{c}\quad-\frac{1}{576} \beta^{4} \bar{\beta}^{*}+\frac{1}{96} \beta^{3} \bar{\beta} \beta^{*}-\frac{1}{24} \beta^{3} \bar{\beta}^{*} \bar{\beta} \\
+\frac{13}{192} \beta^{2} \bar{\beta}^{2} \beta^{*}+\frac{13}{192} \beta^{2} \bar{\beta}^{2} \bar{\beta}^{*}-\frac{1}{24} \beta \bar{\beta}^{3} \beta^{*} \\
+\frac{1}{96} \bar{\beta}^{3} \beta \bar{\beta}^{*}-\frac{1}{576} \bar{\beta}^{4} \beta^{*}-\frac{13}{192} \beta^{* 2} \beta \bar{\beta}^{* 2} \\
+\frac{1}{24} \beta^{* 3} \bar{\beta}^{*} \bar{\beta}-\frac{1}{96} \beta^{* 3} \beta \bar{\beta}^{*}+\frac{1}{576} \beta^{* 4} \bar{\beta} \\
+\frac{1}{24} \beta^{*} \bar{\beta}^{* 3} \beta-\frac{13}{192} \beta^{* 2} \bar{\beta}^{* 2} \bar{\beta}+\frac{1}{576} \bar{\beta}^{* 4} \beta \\
-\frac{1}{96} \bar{\beta}^{* 3} \bar{\beta} \beta^{*}\end{array}$ & $\begin{array}{c}-\frac{1}{576} \beta^{4} \bar{\beta}^{*}+\frac{1}{96} \beta^{3} \bar{\beta} \beta^{*}-\frac{1}{24} \beta^{3} \bar{\beta}^{*} \bar{\beta} \\
+\frac{13}{192} \beta^{2} \bar{\beta}^{2} \beta^{*}+\frac{13}{192} \beta^{2} \bar{\beta}^{2} \bar{\beta}^{*}-\frac{1}{24} \beta \bar{\beta}^{3} \beta^{*} \\
+\frac{1}{96} \bar{\beta}^{3} \beta \bar{\beta}^{*}-\frac{1}{576} \bar{\beta}^{4} \beta^{*}-\frac{13}{192} \beta^{* 2} \beta \bar{\beta}^{* 2} \\
+\frac{1}{24} \beta^{* 3} \bar{\beta}^{*} \bar{\beta}-\frac{1}{96} \beta^{* 3} \beta \bar{\beta}^{*}+\frac{1}{576} \beta^{* 4} \bar{\beta} \\
+\frac{1}{24} \beta^{*} \bar{\beta}^{* 3} \beta-\frac{13}{192} \beta^{* 2} \bar{\beta}^{* 2} \bar{\beta}+\frac{1}{576} \bar{\beta}^{* 4} \beta \\
-\frac{1}{96} \bar{\beta}^{* 3} \bar{\beta} \beta^{*}\end{array}$ & 0 \\
\hline \multirow[t]{2}{*}{6} & $\begin{array}{c}\quad-\frac{1}{13824} \bar{\beta}^{* 6}-\frac{1}{13824} \beta^{6}-\frac{13}{2304} \beta^{3} \beta^{* 2} \bar{\beta}^{*} \\
-\frac{15}{128} \beta^{2} \beta^{* 2} \bar{\beta}^{*} \bar{\beta}-\frac{15}{128} \beta \bar{\beta}^{2} \bar{\beta}^{* 2} \beta^{*}-\frac{13}{2304} \beta \bar{\beta}^{2} \bar{\beta}^{* 3} \\
+\frac{23}{576} \bar{\beta}^{3} \beta^{* 2} \bar{\beta}^{*}-\frac{1}{1152} \beta^{3} \beta^{* 3}-\frac{61}{4608} \beta^{* 4} \bar{\beta}^{* 2}\end{array}$ & $\begin{array}{c}-\frac{1}{13824} \bar{\beta}^{* 6}-\frac{1}{13824} \beta^{6}-\frac{13}{2304} \beta^{3} \beta^{* 2} \bar{\beta}^{*} \\
-\frac{15}{128} \beta^{2} \beta^{* 2} \bar{\beta}^{*} \bar{\beta}-\frac{15}{128} \beta \bar{\beta}^{2} \bar{\beta}^{* 2} \beta^{*}-\frac{13}{2304} \beta \bar{\beta}^{2} \bar{\beta}^{* 3} \\
+\frac{23}{576} \bar{\beta}^{3} \beta^{* 2} \bar{\beta}^{*}-\frac{1}{1152} \beta^{3} \beta^{* 3}-\frac{61}{4608} \beta^{* 4} \bar{\beta}^{* 2}\end{array}$ & \\
\hline & $\begin{array}{c}-\frac{61}{4608} \beta^{4} \bar{\beta}^{2}-\frac{1}{288} \beta^{5} \bar{\beta}-\frac{61}{4608} \beta^{2} \bar{\beta}^{4}+\frac{5}{72} \beta^{* 3} \bar{\beta}^{* 3} \\
+\frac{5}{72} \beta^{3} \bar{\beta}^{3}-\frac{1}{288} \beta^{*} \bar{\beta}^{* 5}-\frac{1}{288} \beta \bar{\beta}^{5}-\frac{61}{4608} \beta^{* 2} \bar{\beta}^{* 4} \\
-\frac{1}{13824} \bar{\beta}^{6}-\frac{13}{2304} \beta^{2} \beta^{* 3} \bar{\beta}-\frac{1}{1152} \bar{\beta}^{3} \bar{\beta}^{* 3} \\
-\frac{1}{288} \beta^{* 5} \bar{\beta}^{*}+\frac{23}{576} \beta^{3} \beta^{*} \bar{\beta}^{* 2}+\frac{23}{576} \beta^{2} \bar{\beta}^{* 3} \bar{\beta} \\
+\frac{23}{576} \beta \bar{\beta}^{2} \beta^{* 3}-\frac{13}{2304} \bar{\beta}^{3} \bar{\beta}^{* 2} \beta^{*}-\frac{1}{13824} \beta^{* 6}\end{array}$ & $\begin{array}{c}-\frac{61}{4608} \beta^{4} \bar{\beta}^{2}-\frac{1}{288} \beta^{5} \bar{\beta}-\frac{61}{4608} \beta^{2} \bar{\beta}^{4}+\frac{5}{72} \beta^{* 3} \bar{\beta}^{* 3} \\
+\frac{5}{72} \beta^{3} \bar{\beta}^{3}-\frac{1}{288} \beta^{*} \bar{\beta}^{* 5}-\frac{1}{288} \beta \bar{\beta}^{5}-\frac{61}{4608} \beta^{* 2} \bar{\beta}^{* 4} \\
-\frac{1}{13824} \bar{\beta}^{6}-\frac{13}{2304} \beta^{2} \beta^{* 3} \bar{\beta}-\frac{1}{1152} \bar{\beta}^{3} \bar{\beta}^{* 3} \\
-\frac{1}{288} \beta^{* 5} \bar{\beta}^{*}+\frac{23}{576} \beta^{3} \beta^{*} \bar{\beta}^{* 2}+\frac{23}{576} \beta^{2} \bar{\beta}^{* 3} \bar{\beta} \\
+\frac{23}{576} \beta \bar{\beta}^{2} \beta^{* 3}-\frac{13}{2304} \bar{\beta}^{3} \bar{\beta}^{* 2} \beta^{*}-\frac{1}{13824} \beta^{* 6} \\
+\frac{5}{384} \beta^{3} \bar{\beta}^{* 3}+\frac{5}{384} \beta^{* 3} \bar{\beta}^{3}-\frac{5}{256} \beta^{2} \beta^{*} \bar{\beta}^{* 2} \bar{\beta} \\
-\frac{5}{256} \beta \beta^{* 2} \bar{\beta}^{*} \bar{\beta}^{2}\end{array}$ & $\begin{array}{c}\frac{5}{384} \beta^{3} \bar{\beta}^{* 3} \\
+\frac{5}{384} \beta^{* 3} \bar{\beta}^{3} \\
-\frac{5}{256} \beta^{2} \beta^{*} \bar{\beta}^{* 2} \bar{\beta} \\
-\frac{5}{256} \beta \beta^{* 2} \bar{\beta}^{*} \bar{\beta}^{2}\end{array}$ \\
\hline
\end{tabular}
required to be present in order to consistently generate

TABLE I. The fourth through sixth orders of $K$ from the Gustavson normal-form calculation and from the FJS procedure are compared. 
higher-order terms in the expansion.

If Table I were continued to higher orders, column 3 would then show differences for each order above 5 . However, if we invoke the flexibility of our procedure to incorporate additional homogeneous $(++C)$ terms at even order as discussed in Sec. VII, we can recapture exactly the Gustavson expansion. Thus if we add difference terms in column 3 to the FJS sixth-order expression, our procedure will then generate the same seventh-order terms as Gustavson. Then the eight-order terms will be different again by $(++C)$ homogeneous terms, which if then added to the FJS eight order will ensure agreement with Gustavson through ninth order and so on.

With our procedure, still other expansions can be generated by adding in combinations of homogeneous $(++C)$ terms other than the ones of Gustavson. And, of course, we are not constrained in our procedure to the use of the Gustavson fourth-order expression (8.3). Any $(++C)$ homogeneous term will work and there are two linearly independent such terms at fourth order.

Thus it is clear that our procedure can generate the normal-form series, but is also capable of producing a whole class of different series for $K$ depending both upon the homogeneous $(++C)$ terms, which are added at even order, and such terms that are used at the first nonvanishing order of the series.

\section{SUMMARY AND DISCUSSION}

The procedure given in Sec. VII provides a direct constructive method for generating a power series in the $\beta$ variables for a formally conserved quantity $K$. We begin at an even order $n_{0}$ (which normally would be taken as 4 since starting at $n_{0}=2$ just reproduces the total energy) with a solution of the homogeneous equation (4.6) having the symmetry $(++C)$. Every odd-order term in the series is determined uniquely by an equation analogous to (6.1) which gives the first odd-order term.

At even orders the problem is more subtle due to the presence of homogeneous solutions to $(4.7)$ when $(n+1)$ is even. In general, (7.5) must hold at a given even order $n_{E}$ in order for a solution to exist at order $\left(n_{E}+2\right)$. At the lowest even order $n_{0}$ there is no problem because (7.1) is automatically true due to Theorem $\mathrm{I}$. At higher even order, the existence of solutions is not automatic. What we have done in Sec. VII is to devise a method of adding homogeneous solutions with $(++D)$ symmetry at order $n_{E}>n_{0}$ in such a way that (7.5) will always be satisfied. The added homogeneous solution is (7.8) is "minimal" and "unique" in the sense that all the terms in it are required in order for (7.5) to be satisfied. The quantity $K$ has $(++D)$ symmetry since each term in the series has that symmetry.

The procedure just described, including the minimal homogeneous $(++D)$ terms to ensure the series continues, can be efficiently implemented using computer algebra systems. Starting with fourth order, we have already been able to generate 24 orders in the conserved quantity $K$, which is twice the number of orders that have been previously generated using other methods [2-4].

It also possible, as discussed, to generate from our pro- cedure a class of expansions for $K$ which result from adding an arbitrary amount of homogeneous $(++C)$ solutions at each even order. We believe that this class includes all possible positive power expansions for a formally conserved quantity $K$. A very important and interesting question is whether certain members of this class of expansions converge better in some sense than others. That question is currently being studied.

\section{APPENDIX}

We discuss here the proofs of the theorems given in Sec. V. All of these theorems involve the application of the $\Omega$ operator (6.3) on solutions of the homogeneous equation (4.8). To facilitate our discussion, it will be useful to rewrite the $D_{1}$ operator of (4.4) as a sum of operators as follows:

$$
D_{1}=\sum_{k= \pm 1, \pm 3} D(k)
$$

where the $D(k)$ are defined by

$$
\begin{aligned}
& D(+3)=\frac{1}{4}\left[\beta^{2} \frac{\partial}{\partial \bar{\beta}^{*}}-\beta^{* 2} \frac{\partial}{\partial \bar{\beta}}\right], \\
& D(-3)=\frac{1}{4}\left[\bar{\beta}^{* 2} \frac{\partial}{\partial \beta}-\bar{\beta}^{2} \frac{\partial}{\partial \beta^{*}}\right], \\
& D(+1)=\frac{1}{4}\left[2 \beta \bar{\beta} \frac{\partial}{\partial \bar{\beta}^{*}}+\beta^{* 2} \frac{\partial}{\partial \beta}-2 \beta^{*} \bar{\beta}^{*} \frac{\partial}{\partial \beta}-\beta^{2} \frac{\partial}{\partial \beta^{*}}\right] \\
& D(-1)=\frac{1}{4}\left[2 \beta^{*} \bar{\beta}^{*} \frac{\partial}{\partial \beta}+\bar{\beta}^{2} \frac{\partial}{\partial \bar{\beta}^{*}}-2 \beta \bar{\beta} \frac{\partial}{\partial \beta^{*}}-\bar{\beta}^{* 2} \frac{\partial}{\partial \bar{\beta}}\right]
\end{aligned}
$$

The $D(k)$ operators act like raising and lowering operators in the sense that when applied to a monomial $M_{p q r s}$ of the form (5.2), the result is a sum of monomials each of which has $k$ more powers of unbarred $\beta$ variables than unbarred variables when compared to the same quantity for the original monomial. More explicitly,

$D_{0} D(k) M_{p q r s}=i(k+p+q-r-s) D(k) M_{p q r s}$.

Using the $D(k)$ operators, it is possible to write the effect of the $\Omega$ operator on solutions of the homogeneous equation (4.8) as follows:

$$
\Omega H=\sum_{k= \pm 1, \pm 3}\left[D(k) D_{0}^{-1} D(-k)\right] H
$$

In (A7) the pairing of the $D(k)$ and $D(-k)$ operators in each term ensures that we have projected out the homogeneous solution as required by the first factor on the right-hand side of (6.3) 
The proof of Theorem I can be accomplished by a straightforward application of the result (A7) with $H=H_{p q}^{++C}$, where the latter is given explicitly in (5.7). The result is that $\Omega H_{p q}^{++C}=0$.
Theorem II states that the operator $\Omega$ states that the operator $\Omega$ satisfies a chain rule when operating on products of solutions of the homogeneous equation. Again we apply (A7):

$$
\begin{aligned}
\Omega\left(H_{1} H_{2}\right)= & \sum_{k= \pm 1, \pm 3} D(k) D_{0}^{-1}\left\{\left[D(-k) H_{1}\right] H_{2}+H_{1}\left[D(-k) H_{2}\right]\right\} \\
= & \sum_{k= \pm 1, \pm 3} D(k) \frac{1}{-i k}\left\{\left[D(-k) H_{1}\right] H_{2}+H_{1}\left[D(-k) H_{2}\right]\right\} \\
= & \sum_{k= \pm 1, \pm 3} \frac{1}{i k}\left\{\left[D(-k) H_{1}\right]\left[D(k) H_{2}\right]+\left[D(k) H_{1}\right]\left[D(-k) H_{2}\right]\right. \\
& \left.\quad+\left[D(k) D(-k) H_{1}\right] H_{2}+H_{1}\left[D(k) D(-k) H_{2}\right]\right\},
\end{aligned}
$$

where we have used (5.4). The first two of the four terms on the right-hand side of (A10) clearly cancel in the sum over $k$. The second two terms just give $\left(\Omega H_{1}\right) H_{2}+H_{1}\left(\Omega H_{2}\right)$, which is the desired result for Theorem II.

Theorem III is proved like Theorem I from a straightforward application of (A7), with $\Omega$ operating on the homogeneous solution (5.11).

[1] M. Hénon and C. Heiles, Astron. J. 69, 73 (1964).

[2] P. Finkler, C. E. Jones, and G. A. Sowell, Phys. Rev. A 42, 1931 (1990).

[3] F. G. Gustavson, Astron. J. 71, 670 (1966).

[4] R. B. Shirts and W. P. Reinhardt, J. Chem. Phys. 77, 5204 (1982).

[5] See, e.g., R. T. Swimm and J. Delos, J. Chem. Phys. 71, 1700 (1979); C. Jaffe and W. P. Reinhardt, ibid. 77, 5191 (1982); and M. Robnik, J. Phys. A 17, 109 (1984).

[6] See, e.g., A. J. Dragt and J. M. Finn, J. Math. Phys. 17, 2215 (1976); 20, 2649 (1979); and L. E. Fried and G. S. Ezra, J. Chem. Phys. 86, 6270 (1987).

[7] S. L. Ziglin, Funct. Anal. Appl. 16, 181 (1983); 17, 6 (1983).

[8] We expect no loss of generality in considering only conserved quantities $K$ that are invariant under time reversal, $x$ reflection, and discrete rotations by multiples of $120^{\circ}$. A theorem of Thompson [G. Thompson, J. Math. Phys. 25, 3474 (1984)] guarantees that $K$ can be picked either even or odd under time reversal. If we have the latter case, we can square $K$ to get a quantity invariant under time reversal. If $K$ is not invariant under the discrete rotations, we can form a new conserved quantity $K^{\prime}$ as follows, which is invariant: $K^{\prime}=K+R K+R^{2} K$, where in $R K, R$ designates the transformation which rotates the $\beta$ variables by $120^{\circ}$ in accordance with (3.3). Should $K^{\prime}$ turn out to be zero, we may sum the squares of each of the three terms on the right-hand side of the above equality to obtain the following nonvanishing conserved quantity: $K^{\prime \prime}=K^{2}+(R K)^{2}$ $+\left(R^{2} K\right)^{2}$. If our conserved quantity is not invariant under $x$ reflections, we can follow a procedure similar to the one just discussed for discrete rotations in order to define a conserved quantity that is invariant under $x$ reflections. Thus if there is a conserved quantity that is not invariant under all the discrete symmetries, it should be possible by a combination of the procedures just outlined to construct one from it that is. To determine whether there exists a conserved quantity in the problem, we then need only consider quantities that are invariant under all the discrete symmetries. 